\title{
Ion Implanted Phosphorous for 4H-SiC VDMOSFETs Source Regions: Effect of the Post Implantation Annealing Time
}

\section{Conference Paper}

Author(s):

Nipoti, Roberta; Parisini, Antonella; Boldrini, Virginia; Vantaggio, Salvatore; Gorni, Marco; Canino, Mariaconcetta; Pizzochero, Giulio; Camarda, Massimo; Woerle, Judith (D); Grossner, Ulrike (D)

Publication date:

2020-07

Permanent link:

https://doi.org/10.3929/ethz-b-000430946

Rights / license:

In Copyright - Non-Commercial Use Permitted

Originally published in:

Materials Science Forum 1004, https://doi.org/10.4028/www.scientific.net/msf.1004.698 


\title{
Ion Implanted Phosphorous for 4H-SiC VDMOSFETs Source Regions: Effect of the Post Implantation Annealing Time
}

\author{
Roberta Nipoti ${ }^{1, a^{*}}$, Antonella Parisini ${ }^{2, b}$, Virginia Boldrini ${ }^{1, c}$, \\ Salvatore Vantaggio ${ }^{2, \mathrm{~d}}$, Marco Gorni ${ }^{2, \mathrm{e}}$, Marica Canino ${ }^{1, \mathrm{f}}$, \\ Giulio Pizzochero ${ }^{1, \mathrm{~g}}$, Massimo Camarda ${ }^{3, \mathrm{~h}}$, Judith Woerle ${ }^{3, \mathrm{i}}$, \\ Ulrike Grossner ${ }^{4, j}$ \\ ${ }^{1}$ CNR-IMM unit of Bologna, Bologna, Italy \\ ${ }^{2}$ University of Parma, DMPCS - CNISM, Parma, Italy \\ ${ }^{3} \mathrm{PSI}$, Villigen, Switzerland \\ ${ }^{4}$ ETH Zurich, Advanced Power Semiconductor Laboratory, Zurich, Switzerland \\ anipoti@bo.imm.cnr.it, bantonella.parisini@fis.unipr.it, cboldrini@bo.imm.cnr.it, \\ dsalvatore.vantaggio@unipr.it, e'gorni.marco@gmail.com, ${ }^{\mathrm{f}}$ canino@bo.imm.cnr.it, \\ gpizzochero@bo.imm.cnr.it, 'hmassimo.camarda@psi.ch, judith.woerle@psi.ch, \\ julrike.grossner@ethz.ch
}

Keyword: $\mathrm{P}$ ion implantation, $\mathrm{Al}$ and $\mathrm{P}$ co-implantation, post implantation annealing, MOSFET

\begin{abstract}
Van der Pauw devices have been fabricated by double ion implantation processes, namely $\mathrm{P}^{+}$and $\mathrm{Al}^{+}$co-implantation. Similarly to the source area in a SiC VD-MOSFET, a $5 \times 10^{18} \mathrm{~cm}^{-3} \mathrm{P}$ plateau is formed on the top of a buried $3 \times 10^{18} \mathrm{~cm}^{-3} \mathrm{Al}$ distribution for electrical isolation from the $\mathrm{n}^{-}$epilayer. The post implantation annealing temperature was $1600{ }^{\circ} \mathrm{C}$. Annealing times equal to $30 \mathrm{~min}$ and $300 \mathrm{~min}$ have been compared. The increase of the annealing time produces both an increase of electron density as well as electron mobility. For comparison a HPSI $4 \mathrm{H}-\mathrm{SiC}$ wafer, $1 \times 10^{20} \mathrm{~cm}^{-3} \mathrm{P}^{+}$ion implanted and $1700{ }^{\circ} \mathrm{C}$ annealed for $30 \mathrm{~min}$ was also characterized.
\end{abstract}

\section{Introduction}

Double ion implantation schedules, one for donor and one for acceptor, are common in the fabrication of semiconductor electronic devices. In the case of vertical MOSFETs such technology allows the fabrication of devices with source and body regions that are both ion implanted. In this case, the source region is co-implanted in the ion implanted body region and the device is called VDMOSFET. 4H-SiC VDMOSFETs are the subject of intense studies.

Most of the studies on the electrical activation of ion-implanted dopants in $4 \mathrm{H}-\mathrm{SiC}$ have used high purity semi-insulating (HPSI) substrates and low-doped epitaxial layers (EPI). Moreover, coimplantation has been studied mostly in the aim to obtain a more efficient electrical doping [1]. In the case of the co-doped source regions of DMOSFETs, the donor type dopant is co-implanted with an acceptor type dopant that, once electrically activated, plays as a compensator center, i.e. it may reduce the n-type doping efficiency.

In ion implanted $4 \mathrm{H}-\mathrm{SiC}$, the commonly used p-type dopant is $\mathrm{Al}$ while the n-type is $\mathrm{P}$. This latter substituted $\mathrm{N}$, because $\mathrm{P}$ shows a higher electrical activation during post implantation annealing at low temperatures, for example $1300{ }^{\circ} \mathrm{C}$ [2]. Moreover, $\mathrm{P}$ does not suffer of the phenomenon of auto-compensation that $\mathrm{N}$ shows for implanted concentrations $\geq(2-5) \times 10^{19} \mathrm{~cm}^{-}$ 3 [3]. In the case of $\mathrm{n}$-channel vertical $4 \mathrm{H}-\mathrm{SiC}$ DMOSFET, the p-type body is, usually, $\mathrm{Al}$ implanted with concentration in the $10^{17}-10^{18}$ decades while the n-type source is, usually, $\mathrm{P}$ implanted with concentration in the high $10^{18} \mathrm{~cm}^{-3}$ decade. The requirements of optimal electrical activation and optimal lattice recovery in the $\mathrm{Al}$ ion implanted channel region, impose that the post implantation annealing temperature is at least $1600{ }^{\circ} \mathrm{C}$. 
In this work, the electrical activation of $\mathrm{P}$ co-implanted with $\mathrm{Al}$ has been studied for double ion implantation schedules identical to those used for DMOSFET fabrication. The post implantation annealing temperature of $1600{ }^{\circ} \mathrm{C}$ is used and the post implantation annealing time has been $30 \mathrm{~min}$, a commonly used processing time, and $300 \mathrm{~min}$ that is a long annealing time, rarely used.

The aim of this study is to demonstrate that van der Pauw devices, emulating the doping configuration of the DMOSFET source, can be used to study the $\mathrm{P}$ electrical activation in the identical constrain as a real DMOSFET source. This should let us look for a possible effect of Al compensation, and for an effect of the annealing time, if any. The latter items are suggested by the fact that long annealing times have been seen to be relevant in the case of $\mathrm{Al}$ ion implanted $4 \mathrm{H}-$ $\mathrm{SiC}[4,5]$.

\section{Experimental}

A n-type $4 \mathrm{H}-\mathrm{SiC}$ wafer $4^{\circ}$ off $<0001>$ axis, homo epitaxial, with $3 \times 10^{15} \mathrm{~cm}^{-3}$ net donor concentration in the epi-layer was used for this study. $\mathrm{P}^{+}$ion implanted regions next to the wafer surface, with a cloverleaf van der Paw (vdP) geometry and within an $\mathrm{Al}^{+}$ion implanted well, were obtained by two selected area ion implantation processes. The implantation temperature was $400{ }^{\circ} \mathrm{C}$. To obtain an almost flat $\mathrm{P}$ plateau next to the wafer surface, the $\mathrm{P}^{+}$ion implantation schedule was with multiple energies and different doses through a $\mathrm{SiO}_{2}$ layer. To obtain a buried $\mathrm{Al}$ depth profile, the implantation schedule was a single high energy $\mathrm{Al}^{+}$ion implantation process, through the same $\mathrm{SiO}_{2}$ layer. The ion implantation mask was a thick Al film. Fig. 1 shows the simulated $\mathrm{P}$ and $\mathrm{Al}$ depth profiles by the SRIM code [6]. The $\mathrm{P}$ depth profile has a plateau of about $5 \times 10^{18} \mathrm{~cm}^{-3}$ and a full width half maximum (FWHM) of about $225 \mathrm{~nm}$. The Al depth profile has a buried peak about $3 \times 10^{18} \mathrm{~cm}^{-3}$ in height at around $390 \mathrm{~nm}$ in depth, which smoothly decreases towards the wafer surface where it reaches a concentration of about $2 \times 10^{17} \mathrm{~cm}^{-3}$. The in-depth and lateral electrical isolation of the n-type $\mathrm{vdP}$ will depend on $\mathrm{P}$ and $\mathrm{Al}$ electrical activation, on the partial ionization of the $\mathrm{P}$ donors and the $\mathrm{Al}$ acceptors at the temperatures of electrical measurements, and on the device geometry. In Fig. 1, the estimated distance between the two metallurgical junctions, one at the cross point between $\mathrm{P}$ and $\mathrm{Al}$ depth profiles, and one at the cross point between $\mathrm{Al}$ depth profile and n-type substrate, is about $350 \mathrm{~nm}$. Each vdP device has square symmetry with a central area of $500 \mu \mathrm{m}$ side that is connected to four leaves by $50 \times 50 \mu \mathrm{m}^{2}$ large bridges (one per leaf). Each leaf has rectangular shape and it is positioned parallel to the vdP side at $50 \mu \mathrm{m}$ distance. The vdP leaves have to remain electrically isolated from the central area of the $\mathrm{vdP}$, except for the bridge regions, thanks to the $\mathrm{Al}$ ion implanted well, whose as-implanted value near the surface is $2 \times 10^{17} \mathrm{~cm}^{-3}$. Finally, the whole vdP structure has to remain laterally isolated from the ntype epilayer because the Al implanted well extends $50 \mu \mathrm{m}$ far away from the external border of a square region defined by the four leaves. Hypothesizing that ion implanted $\mathrm{Al}$ and $\mathrm{P}$ may be 40-100\% electrically active, by neglecting the presence of deep levels, a rough estimation of the $p$-n junction depletion width for abrupt and semi-infinite doping distribution is between 1 and $3 \mu \mathrm{m}$ and falls mostly on the lower doping side of the p-n junction. Next to the sample surface in lateral direction, the depletion region extends in the $\mathrm{p}$ base on the vdP side, while it extends in the $\mathrm{p}$ base or in the $\mathrm{n}^{-}$epilayer depending on the temperature of measurements on the border of the $p$ well. In all the

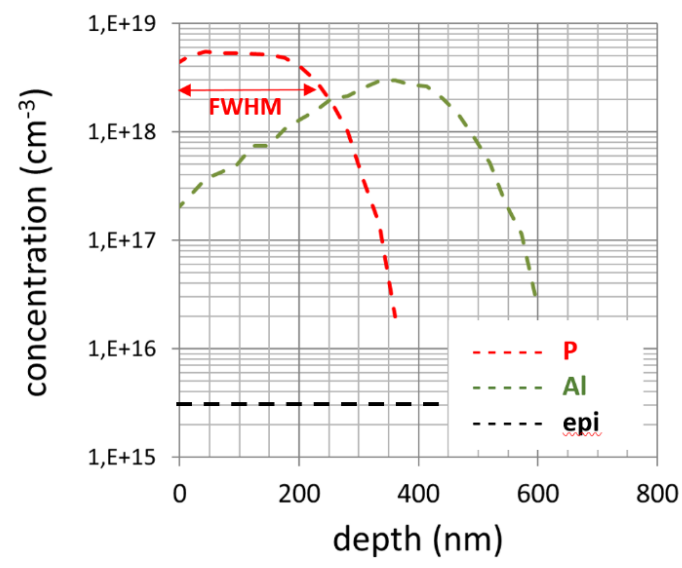

Fig.1. SRIM [6] simulations of the P and Al ion implanted depth profiles in the specimens of this study. The net donor concentration of the epi-layer (epi) and the full width at half maximum (FWHM) of the $\mathrm{P}$ profile are also shown. 
cases, the used $50 \mu \mathrm{m}$ lateral quotes guarantee the lateral electrical isolation of the vdP device geometry. Along the vertical direction, the $350 \mathrm{~nm}$ thick p-type body region is fully depleted, because of its low thickness. The fact the resistance of this depleted layer is always much higher than that of the top P implanted layer, is a guarantee that during electrical measurements the current flows only in the P implanted thickness of the vdP device.

Post implantation annealing was done at $1600{ }^{\circ} \mathrm{C}$, in high purity Ar at atmospheric pressure, with a carbon film coating the implanted surface (C-cap) [7], and two annealing times: $30 \mathrm{~min}$ and $300 \mathrm{~min}$. Ni ohmic contacts were fabricated on the leaves of the vdP devices.

For comparison, a $\mathrm{P}$ box profile of $1 \times 10^{20} \mathrm{~cm}^{-3}$ height $300 \mathrm{~nm} \mathrm{FWHM,} \mathrm{obtained} \mathrm{in} \mathrm{a} \mathrm{high} \mathrm{purity}$ semi-insulating (HPSI) 4H-SiC sample by ion implantation and annealing at $1700{ }^{\circ} \mathrm{C}$ for 5 min with C-cap in high purity Ar at atmospheric pressure, has been also studied. In this case, the vdP device is a square with $5 \mathrm{~mm}$ side and triangular Ni contacts on the corners.

Table I summarizes the samples of this study. Labels A and B are used for the vdP devices on the $4 \mathrm{H}-\mathrm{SiC}$ epitaxial wafer (EPI), $30 \mathrm{~min}$ and $300 \mathrm{~min}$, respectively, while, label $\mathrm{C}$ is used for the vdP device on the $4 \mathrm{H}-\mathrm{SiC}$ HPSI wafer.

Sheet resistance and Hall-effect measurements on the vdP devices were performed in the temperature range $70-640 \mathrm{~K}$. In this range, the sign of the Hall coefficient was always congruent with a dominant electron current conduction; moreover, the vdP devices on the EPI substrate resulted laterally isolated from the nearest devices. This let us assume that the electrical isolation between the leaves of the central area of the vdP devices is appropriate for reliable electrical characterizations. Sheet resistance times the FWHM value of the ion implanted P depth profile provides an estimate of the material resistivity in the ion-implanted layer. The ratio between the measured Hall coefficient and the measured sheet resistance allows us to measure the free carrier mobility in the ion-implanted layer. Due to the use of different experimental set-ups for Hall-effect measurements, the magnetic field was $0.8 \mathrm{~T}$ and $1 \mathrm{~T}$ for measurement temperatures lower and higher than $300 \mathrm{~K}$, respectively. No difference between the $300 \mathrm{~K}$ data at different magnetic field was detected.

Table I. Samples labels, processing parameters, and estimated $\mathrm{P}$ and $\mathrm{Al}$ electrical activations

\begin{tabular}{|c|c|c|c|c|c|c|c|}
\hline \multirow{2}{*}{$\begin{array}{l}\text { Sample } \\
\text { label }\end{array}$} & \multirow{2}{*}{$\begin{array}{l}4 \mathrm{H}-\mathrm{SiC} \\
\text { wafer }\end{array}$} & \multirow{2}{*}{$\begin{array}{c}\text { as-impl. } \\
\mathrm{P} \\
{\left[\mathrm{cm}^{-3}\right]}\end{array}$} & \multirow{2}{*}{$\begin{array}{c}\text { as-impl. } \\
\mathrm{Al} \\
{\left[\mathrm{cm}^{-3}\right]}\end{array}$} & \multicolumn{2}{|c|}{$\begin{array}{l}\text { Post implantation } \\
\text { annealing }\end{array}$} & \multirow{2}{*}{$\begin{array}{c}\text { P electrical } \\
\text { activation } \\
{[\%]}\end{array}$} & \multirow{2}{*}{$\begin{array}{c}\text { Al electrica } \\
\text { activation }^{\text {(\# }} \\
{[\%]}\end{array}$} \\
\hline & & & & $\begin{array}{c}\text { Temperature } \\
{\left[{ }^{\circ} \mathrm{C}\right]}\end{array}$ & $\begin{array}{l}\text { Time } \\
{[\mathrm{min}]}\end{array}$ & & \\
\hline A & EPI & $5 \times 10^{18}$ & $3 \times 10^{18}$ & 1600 & 30 & $\geq 51$ & 81 \\
\hline B & EPI & $5 \times 10^{18}$ & $3 \times 10^{18}$ & 1600 & 300 & $\geq 57$ & 77 \\
\hline C & HPSI & $1 \times 10^{20}$ & ----- & 1700 & 5 & $\geq 61$ & ----- \\
\hline
\end{tabular}

(\#) electrical activation for identically $\mathrm{Al}^{+}$ion implanted and post implantation annealed body regions from ref. [5]

\section{Results}

The comparisons of the temperature dependence of the resistivity, the Hall electron density, and the Hall mobility of the samples of this study in the temperature range $70-680 \mathrm{~K}$, are compared in Figs. 2(a,b,c), respectively. In Figs. 2(a) and 2(b), the conversion from measured data to material parameters uses the values of the full width at half-maximum (FWHM) of the SRIM depth profiles (see Fig. 1).

In Fig. 2(a), sample A shows the highest resistivity and sample $\mathrm{C}$ the lowest. However, while sample $\mathrm{C}$ shows a positive temperature coefficient over the whole temperature range, samples $\mathrm{A}$ and $\mathrm{B}$ show a positive temperature coefficient (resistivity decreasing with decreasing temperature) from $640 \mathrm{~K}$ down to around $400-420 \mathrm{~K}$ and move to a negative temperature coefficient 
(resistivity increasing with decreasing temperature) for further decreasing temperatures down to $70 \mathrm{~K}$.

In Fig. 2(b), the carrier density data increase progressively going from sample A, to B, and to $\mathrm{C}$, at any temperature, with a common feature trend. In fact, all the curves show a positive temperature coefficient in the high temperature region, go through a minimum, and show a negative temperature coefficient for further decreasing temperature down to $70 \mathrm{~K}$. These curve minima fall at higher temperatures going from sample A to B to C, i.e. with increasing electron density. Finally, in the case of sample $\mathrm{C}$, carrier concentration seems to approach a saturation value at the lowest measurement temperature.

In Fig. 2(c), all the mobility curves show the typical bell shape trend, with a maximum around 200-300 K and decreasing values towards the lower and the higher temperatures of measurement. In the lower temperature region, the mobility data increase going from sample $\mathrm{A}$ to $\mathrm{B}$ to $\mathrm{C}$, while in the higher temperature region the mobility data are higher going from sample $\mathrm{C}$ to $\mathrm{A}$ to $\mathrm{B}$, the two latter also showing the maximum at about the same T.

A reliable estimation of the electrically activated fraction of the ion implanted $\mathrm{P}$, could be obtained by the simultaneous fitting of correspondent Hall electron density and Hall mobility curves in Figs. 2(b,c) by using the neutrality equation and the appropriate mobility model in the temperature region above the curve minimum and maximum, respectively. However, for preliminary comments, here we decided to assume a Hall factor equal to 1 and to roughly estimate the value of the $\mathrm{P}$ donor density by taking it equal or major than the Hall carrier density at the maximum measurement temperature. The so obtained values are in Table I.

The results of the study on the electrical activation of $\mathrm{Al}$ in the ion implanted body region are available in ref. [5] and are shown in Table I too.

\section{Discussion}

Because in samples A and B the high-temperature Hall density resulted in a lower-than- critical
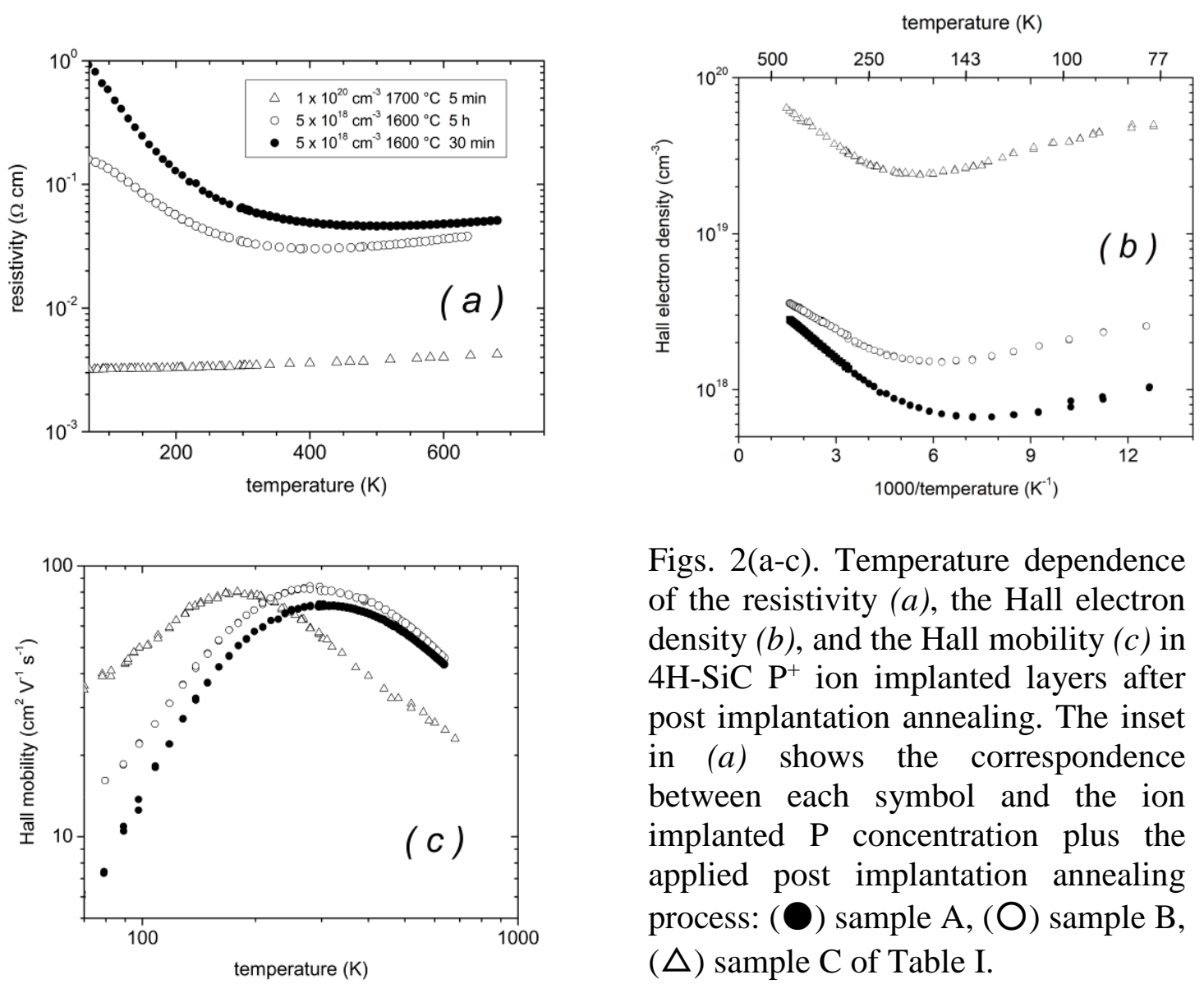

Figs. 2(a-c). Temperature dependence of the resistivity $(a)$, the Hall electron density $(b)$, and the Hall mobility $(c)$ in $4 \mathrm{H}-\mathrm{SiC} \mathrm{P}^{+}$ion implanted layers after post implantation annealing. The inset in (a) shows the correspondence between each symbol and the ion implanted $\mathrm{P}$ concentration plus the applied post implantation annealing process: $(\mathbf{O})$ sample $\mathrm{A},(\mathrm{O})$ sample $\mathrm{B}$, $(\triangle)$ sample $\mathrm{C}$ of Table I. 
density for the Mott transition and significantly lower than the effective density of states (EDOS) in the conduction band (CB), a carrier freezing into impurity states is expected and the Boltzmann statistics can be used to describe the occupancy of the electronic states. The high doping level, however, certainly, leads to a significant reduction of the thermal ionization energy of the donors with respect to the insulated impurity, and induces the formation of an impurity band. In this frame, the concave shape of the Hall electron density curves of Fig. 2(b) stands for transition from transport into extended states to transport into localized states, or at least transport with a significant contribution of the latter mechanism, when the temperature decreases. Though for sample $\mathrm{C}$ the electron gas should be degenerate (depending on $\mathrm{T}$ ), the strong similarity of the trends suggests to extend the same hypothesis to the curve $\mathrm{C}$. Consequently, the shift of the curve minima towards lower temperature is congruent with the increase of the electron density going from sample A to B and to $\mathrm{C}$, as expected for this phenomenology.

Transport into localized states is favored by compensation, which is unavoidable in semiconductors regions that have been doped by ion implantation. In the case of the A and B samples of this study, an additional compensation effect due to the co-implanted Al acceptors need to be considered. By knowing that the electrical activation of the implanted $\mathrm{Al}$ in the body region is about $80 \%$ [5] and by assuming a similar electrical activation for $\mathrm{Al}$ in the $\mathrm{Al}$ and $\mathrm{P}$ coimplanted region, we can hypothesize that the $\mathrm{P}$ donor compensation by $\mathrm{Al}$ acceptors is significant next to the metallurgical junction. However, it sharply decreases going towards the sample surface because of the $\mathrm{Al}$ and $\mathrm{P}$ depth profiles (Fig. 1). Obviously, the electron density curves of Fig. 2(b) are average values over the $\mathrm{P}$ implanted thickness.

The positive temperature coefficient of all the resistivity curves of Fig. 2(a) in the high temperature region leads to the conclusion that in spite of the increasing electron density with increasing temperature, the decrease of the electron mobility (due to the increasing phonon scattering) has a dominant effect. In the low temperature region, the still positive temperature coefficient of the resistivity curve of sample $\mathrm{C}$ is consistent with a metal-like transport, as mentioned above. Differently, in the same temperature region, the negative temperature coefficient of the resistivity curves of samples A and B is a further indication of a semiconductor behavior.

The discussion on the mobility curves needs a distinction between sample $\mathrm{C}$ and samples $\mathrm{A}$ and $\mathrm{B}$, and between low and high temperature regions. In the low temperature region, the electron mobility increases with the increasing of the electron density from sample A to B to C (Fig. 2(b)). This is justified by a transport involving localized states (increasing the doping level increases the hopping probability) and by the approaching of the metallic conduction with the increasing of the electron density (the mean energy of the electron gas is enhanced by approaching degenerate conditions). In the high temperature region, electron mobility in sample $C$ is lower than in sample $\mathrm{A}$, and this latter mobility is lower than in sample B. In the temperature region dominated by the phonon scattering, a decrease of carrier mobility with increasing dopant density has been seen in the case of p-type $4 \mathrm{H}-\mathrm{SiC}$ when the doping concentration is very high [8]. We hypothesize that in the case of sample $\mathrm{C}$ the very high doping level is responsible of the lower mobility values in the high temperature region, and with respect to the values of mobility in samples A and B. The effect is expected be related to the different statistics of the electron gas in samples A, B with respect to sample $\mathrm{C}$, and also to the stronger contribution of the Coulomb scattering in $\mathrm{C}$ sample, very effective notwithstanding the enhanced phonon scattering at high temperatures. An appropriate model to justify this trend is under construction. The mobility curves of samples A and B in the high temperature region are very close in values, which may be justified by a very similar donor level, i.e. very similar lattice and thus very similar phonon scattering in the two samples.

Looking at the electron density and mobility curves of samples A and B over the whole temperature range, it is evident that for identical ion implantation process and post implantation annealing temperature of $1600{ }^{\circ} \mathrm{C}$, the increase of the annealing time from $30 \mathrm{~min}$ to $300 \mathrm{~min}$ leads to a contemporaneous increase of electron density and electron mobility. The mobility increase can be justified by a decreasing of the scattering centers with the increasing of annealing time. The contemporaneous electron density increase could not be justified by a sole increase of the P 
electrical activation because this would increase the density of the Coulomb scattering centers and thus would produce a mobility reduction. On the contrary, the electron density increase can be justified by a sole decrease of the compensation centers, which, being fully ionized, also contributes to the density of scattering centers. Obviously, an increased P electrical activation with increasing annealing time at constant annealing temperature cannot be excluded. In such a case, the reduction of compensation center has to overcome the increase of donors. Quantitative estimation will be possible only when the code for the simulation of carrier and mobility curves will be available.

The electron density, mobility and resistivity data at room temperature obtained by the $\mathrm{P}$ and Al co-implanted vdP are in agreement with the literature data for equally implanted $\mathrm{P}$ concentration and equal post implantation annealing temperature. This excludes any significant contribution of the $\mathrm{Al}$ acceptor compensation in the DMOSFETs source region.

Comparing the observed effect of the annealing time with literature data has not been possible because the processing condition of the samples varied significantly.

\section{Conclusions}

This study shows that vdP devices can be included in the layout of DMOSFETs fabrication for the qualification of the electron transport in the device source region. This study evidences also that in the $\mathrm{P}$ and $\mathrm{Al}$ co-implanted source region the acceptor compensation is negligible. Finally, this study shows a slight positive effect of the increasing annealing time on the electron transport in the source regions (higher electron density and higher electron mobility). This may not be relevant for the actual DMOSFET performance, but it is of high interest for a better understanding of the post implantation annealing thermodynamics in $4 \mathrm{H}-\mathrm{SiC}$.

\section{Acknowledgments}

The assistance of Mr. Michele Bellettato, Mr. Michele Sanmartin, and Mr. Filippo Bonafè during sample processing is acknowledged.

\section{References}

[1] S. Blanqué, J. Lyonnet, R. Pérez, P. Terziyska, S. Contreras, P. Godignon, N. Mestres, J. Pascual, and J. Camassel, Full wafer size investigation of $\mathrm{N}^{+}$and $\mathrm{P}^{+}$co-implanted layers in $4 \mathrm{H}-$ SiC, phys. stat. sol. (a) 202 (2005) 698-704.

[2] M.A. Capano, R. Santhakumar, R. Venugopal, M.R. Melloch, and J.A. Cooper, Jr, Phosphorus Implantation into 4H-Silicon Carbide, J. Electr. Mater. 29 (2000) 210-214.

[3] M. Laube, F. Schmid, G. Pensl, G. Wagner, M. Linnarsson, M. Maier, Electrical activation of high concentrations of N and P ions implanted into 4H-SiC, J. Appl. Phys. 92 (2002) 549-554.

[4] R. Nipoti, A. Carnera, G. Alfieri, L. Kranz, About the electrical activation of $1 \times 10^{20} \mathrm{~cm}^{-3}$ ion implanted $\mathrm{Al}$ in $4 \mathrm{H}-\mathrm{SiC}$ at annealing temperatures in the range $1500-1950^{\circ} \mathrm{C}$, Mater. Sc. Forum 924 (2018) 333-338.

[5] R. Nipoti, A. Parisini, V. Boldrini, S. Vantaggio, M. C. Canino, M. Sanmartin, and G. Alfieri, $3 \times 10^{18}-1 \times 10^{19} \mathrm{~cm}^{-3} \mathrm{Al}^{+}$ion implanted $4 \mathrm{H}-\mathrm{SiC}$ : annealing time effect, accepted for presentation at ICSCRM2019, Poster Mo-P-31.

[6] SRIM 2008, http://www.srim.org/

[7] R. Nipoti, F. Mancarella, F. Moscatelli, R. Rizzoli, S. Zampolli, M. Ferri, Carbon-Cap for Ohmic Contacts on Ion-Implanted 4H-SiC, Electrochem. Solid-Sate Lett. 13 (2010) H432-H435.

[8] A. Parisini, and R. Nipoti, Analysis of the hole transport through valence band states in heavy Al doped 4H-SiC by ion implantation, J. Appl. Phys. 114 (2013) 243703. 